\title{
ON A CONJECTURE OF FOMIN
}

\author{
ROY L. ADLER
}

Recently P. R. Halmos [5] mentioned a question raised by S. V. Fomin as to whether it follows that two measure preserving transformations are conjugate if they are unitarily equivalent and possess the same entropy. The purpose of this work is to concoct a counterexample to this conjecture.

Let $X$ be the unit interval with Borel measurability and Lebesgue measure. Let $X \times X$ be the unit square and $X \times X \times X$ the unit cube with the usual direct product measurabilities and measures. Finally let $T_{\gamma}, T_{\gamma, \alpha}$, and $T_{\gamma, \alpha, \phi}$ denote the following measure preserving transformations defined respectively on the unit interval, unit square, and unit cube by

$$
\begin{aligned}
T_{\gamma}(x) & =x+\gamma, \\
T_{\gamma, \alpha}(x, y) & =(x+\gamma, y+\alpha(x)), \\
T_{\gamma, \alpha, \phi}(x, y, z) & =(x+\gamma, y+\alpha(x), \phi z)
\end{aligned}
$$

where $\gamma$ is a real number, $\alpha$ is a real-valued measurable function on the unit interval, all additions are modulo 1 , and $\phi$ is a measure preserving transformation on the unit interval.

The entropy $h\left(T_{\gamma}\right)=0$ can be found explicitly calculated in [3] (for the definition of entropy of a measure preserving transformation see [3] or [7]). Abramov [1] has shown $h\left(T_{\gamma, \alpha}\right)=h\left(T_{\gamma}\right)$. By a result on the entropy of direct product transformations [7] we have $h\left(T_{\gamma, \alpha, \phi}\right)$ $=h\left(T_{\gamma, \alpha}\right)+h(\phi)$. Anzai [2] in his extensive examination of skew product transformations of the type $T_{\gamma, \alpha}$ has shown that the ergodic measure preserving transformations $T_{\gamma, \alpha_{m}}$ and $T_{\gamma, \alpha_{n}}$ with $\gamma$ irrational, $\alpha_{m}(x)=m x$ and $\alpha_{n}(x)=n x$, and $|n| \neq|m|$ are not conjugate although they are unitarily equivalent. Clearly both have entropy zero and supply an immediate counterexample to Fomin's question. However, if strictly positive entropy be required, we must be slightly more elaborate. Let $\phi$ be the "two-shift" on the unit interval (for the definition see $\left[4\right.$, p. 8]). Consider $T_{\gamma, \alpha_{m}, \phi}$ and $T_{\gamma, \alpha_{n}, \phi}$ with $\gamma$ irrational and $|n| \neq|m|$. These transformations are unitarily equivalent because they are direct products of pairwise unitarily equivalent transformations. Furthermore $h\left(T_{\gamma, \alpha_{m}, \phi}\right)=h\left(T_{\gamma, \alpha_{n}, \phi}\right)=h(\phi)=\log 2$ (the entropy of the "two-shift" can be found calculated in [3]). The subsequent analysis will show that $T_{\gamma, \alpha_{m}, \phi}$ is not conjugate to $T_{\gamma, \alpha_{n}, \phi}$.

Received by the editors March 30, 1961. 
Suppose $R$ is a measure preserving transformation which provides an isomorphism between $T_{\gamma, \alpha_{m}, \phi}$ and $T_{\gamma, \alpha_{n}, \phi}$ : i.e., $R T_{\gamma, \alpha_{m}, \phi} R^{-1}=T_{\gamma, \alpha_{n}, \phi}$ a.e. If we represent the transformation $R$ as follows

$$
R(x, y, z)=(f(x, y, z), g(x, y, z), h(x, y, z))
$$

where $f, g$, and $h$ are measurable functions defined on the unit cube with values in the unit interval, we get the relations

(i) $f(x, y, z)+\gamma=f(x+\gamma, y+m x, \phi z) \quad(\bmod 1)$ a.e.,

(ii) $g(x, y, z)+n f(x, y, z)=g(x+\gamma, y+m x, \phi z)(\bmod 1)$ a.e.,

(iii) $h(x, y, z)=h(x+\gamma, y+m x, \phi z)$ a.e.

From (i) it is easy to see that the function $e^{2 \pi i f}$ is a proper function of the transformation $T_{\gamma, \alpha_{m}, \phi}$ corresponding to the proper value $e^{2 \pi i \gamma}$. But so is the function whose value at $(x, y, z)$ is $e^{2 \pi i x}$. Since an ergodic transformation can only have simple proper values (see $[4$, p. 34]), we have, for some $u$,

$$
e^{2 \pi i f(x, y, z)}=e^{2 \pi i(x+u)} \quad \text { a.e. }
$$

Thus

$$
f(x, y, z)=x+u(\bmod 1) \quad \text { a.e. }
$$

Next let us determine $g(x, y, z)$. We can expand the function $e^{2 \pi i o}$ in its Fourier series

$$
e^{2 \pi i g(x, y, z)} \sim \sum_{p, q} g_{p, q}(z) e^{2 \pi i(p x+q y)}
$$

where

$$
g_{p, q}(z)=\int_{X} \int_{X} \exp [2 \pi i(g(x, y, z)-p x-q y)] d x d y \quad \text { a.e. }
$$

Consequently

$$
g_{p, q}(\phi z)=\int_{X} \int_{X} \exp [2 \pi i(g(x, y, \phi z)-p x-q y)] d x d y \quad \text { a.e. }
$$

Since $T_{\gamma, \alpha_{m}}$ is measure preserving on the square, the integral remains unchanged after applying this transformation to the function being integrated; i.e.,

$$
\begin{aligned}
g_{p, q},(\phi z)=\int_{X} \int_{X} \exp [2 \pi i(g(x+\gamma, y+m x, \phi z)-p(x+\gamma) & \\
-q(y+m x))] d x d y & \text { a.e. }
\end{aligned}
$$

Utilizing relation (ii) and the above calculated value of $f(x, y, z)$ we obtain 


$$
\begin{aligned}
g_{p, q}(\phi z)= & \exp [2 \pi i(n u-p \gamma)] \int_{X} \int_{X} \\
& \cdot \exp [2 \pi i(g(x, y, z)+(n-p-m q) x-q y)] d x d y \quad \text { a.e. }
\end{aligned}
$$

or

$$
g_{p, q}(\phi z)=\exp [2 \pi i(n u-p \gamma)] g_{p-(n-m q), q}(z) \quad \text { a.e. }
$$

From this results

$$
\left|g_{p-k(n-m q), q}(z)\right|=\left|g_{p, q}\left(\phi^{k} z\right)\right| \quad \text { a.e. }
$$

If $g_{p, q}$ does not vanish almost everywhere, there exists a $\delta>0$ such that the set $A_{p, q}=\left\{z:\left|g_{p, q}(z)\right| \geqq \delta\right\}$ has strictly positive measure. By the recurrence theorem [4, p. 10] almost all $z$ in $A_{p, q}$ return an infinite number of times to the set $A_{p, q}$ under successive applications of $\phi$. This means that for each $z$ in set of nonzero measure $\left|g_{p, q}\left(\phi^{k} z\right)\right| \geqq \delta$ for an infinite number of $k$. Thus if $n-m q \neq 0$ it follows from (**) that for $z$ in a set of nonzero measure an infinite number of the Fourier coefficients in the expansion $\left(^{*}\right)$ have absolute value greater than or equal to $\delta$. This contradicts the square summability of the coefficients in the Fourier series for almost all $z$. Hence $g_{p, q}(z)=0$ a.e., if $q \neq n / m$. For $q=n / m$ we have

$$
g_{p, n / m}(\phi z)=\exp [2 \pi i(n u-p \gamma)] g_{p, n / m}(z) \quad \text { a.e. }
$$

As is well known the "two-shift" is strongly mixing which implies that the only proper functions are constants (see [4, pp. 37, 39]). Thus

$$
g_{p, n / m}(z)=g_{p}=\text { constant } \quad \text { a.e. }
$$

We then have

$$
\begin{aligned}
\exp [2 \pi i g(x, y, z)] & \sim \sum_{p} g_{p} \exp [2 \pi i(p x+n y / m)] \\
& \sim \exp [2 \pi i(\theta(x)+n y / m)]
\end{aligned}
$$

where $\theta$ is some real-valued measurable function of $x$. So

$$
g(x, y, z)=\theta(x)+n y / m(\bmod 1) \quad \text { a.e. }
$$

For $R$ to be measure preserving it is clear that $n / m= \pm 1$ which contradicts the assumption that $|n| \neq|m|$. Therefore $T_{\gamma, \alpha_{m}, \phi}$ is not conjugate to $T_{\gamma, \alpha_{n}, \phi}$.

As we have seen above Fomin's question has in general a negative answer. The corresponding problem restricted to shift transformations is still unsettled. That is, given two shifts having the same 
entropy but based on different measure spaces containing a finite number of points and nonuniformly distributed measures we know they are unitarily equivalent. But are they conjugate? Halmos [5] has suggested that probably the answer is no. But one particular case has been examined which indicates the answer may be yes! Meshalkin [6] apparently has produced a measure preserving transformation which supplies an isomorphism between one shift based on a space of four points with measures $1 / 4,1 / 4,1 / 4,1 / 4$ and another based on a space of five points with measures $1 / 8,1 / 8,1 / 8,1 / 8,1 / 2$.

\section{REFERENCES}

1. L. M. Abramov, On entropy of flows, Dokl. Akad. Nauk SSSR 128 (1959), 873-875.

2. H. Anzai, Ergodic skew product transformations on the torus, Osaka Math. J. 3 (1951), 83-99.

3. P. R. Halmos, Entropy in ergodic theory, Univ. of Chicago, Chicago, Ill., 1959.

4. - Lectures on ergodic theory, Math. Soc. of Japan, 1956.

5. - Recent progress in ergodic theory, Bull. Amer. Math. Soc. 67 (1961), $70-80$

6. L. D. Meshalkin, A case of isomorphism of Bernoulli schemes, Dokl. Akad. Nauk SSSR 128 (1959), 41-44.

7. V. A. Rokhlin, Entropy of metric automorphism, Dokl. Akad. Nauk SSSR 124 (1959), 768-771.

iBM Research Center, Yorktown Heights, New York 